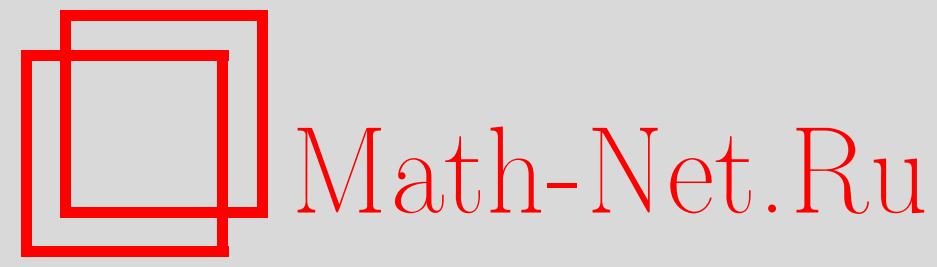

F. Götze, A. N. Tikhomirov, Limit theorems for spectra of random matrices with martingale structure, Теория вероятн. и ее примен., 2006, том 51, выпуск 1, 171-192

DOI: https://doi.org/10.4213/tvp153

Использование Общероссийского математического портала MathNet.Ru подразумевает, что вы прочитали и согласны с пользовательским соглашением

http://www. mathnet.ru/rus/agreement

Параметры загрузки:

IP : 18.234 .156 .22

26 апреля 2023 г., 17:43:33

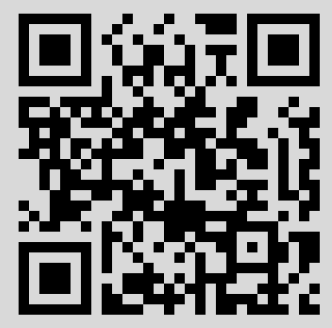




\title{
LIMIT THEOREMS FOR SPECTRA OF RANDOM MATRICES WITH MARTINGALE STRUCTURE ${ }^{1)}$
}

\begin{abstract}
Мы изучаем классический ансамбль вещественных симметричных случайных матриц, введенный Ю. Вигнером. В работе развивается метод Ч. Стейна для изучения аспмптотики ожидаемой спектральной функции распределения матриц большой размерности. Метод основан на дифференциальном уравнении, которому удовлетворяет плотность полукругового закона.
\end{abstract}

Ключевые слова и фразы: случайные матрицы, метод Стейна, полукруговой закон.

1. Introduction. Let $X_{j k}, 1 \leqslant j \leqslant k<\infty$, be triangular array of random variables with $\mathbf{E} X_{j k}=0$ and $\mathbf{E} X_{j k}^{2}=\sigma_{j k}^{2}$, and let $X_{k j}=X_{j k}$, for $1 \leqslant j<k<\infty$. For a fixed $n \geqslant 1$, denote by $\lambda_{1} \leqslant \cdots \leqslant \lambda_{n}$ the eigenvalues of the symmetric $n \times n$ matrix

$$
\mathbf{W}=(W(j, k))_{j, k=1}^{n}, \quad W(j, k)=n^{-1 / 2} X_{j, k}, \quad \text { for } \quad 1 \leqslant j \leqslant k \leqslant n,
$$

and define its empirical spectral distribution function by

$$
F_{n}(x)=\frac{1}{n} \sum_{j=1}^{n} I_{\left\{\lambda_{j} \leqslant x\right\}},
$$

where $I_{B}$ denotes the indicator of an event $B$. We investigate the convergence of the expected spectral distribution function $\mathbf{E} F_{n}(x)$ to the distribution function of Wigner's semicircle law.

Let $g(x)$ and $G(x)$ denote the density and the distribution function of the standard semicircle law, that is,

$$
g(x)=\frac{1}{2 \pi} \sqrt{4-x^{2}} I_{\{|x| \leqslant 2\}}, \quad G(x)=\int_{-\infty}^{x} g(u) d u .
$$

* Fakultät für Mathematik, Universität Bielefeld, Postfach 100131, 33501 Bielefeld 1, Germany; e-mail: goetze@math.uni-bielefeld.de

** Сыктывкарский государственный университет, Октябрьский пр., 55, 167001 Сыктывкар, Россия; e-mail: tikhomir@math.uni-bielefeld.de

1) Reasearch was supported by the DFG-Forschergruppe FOR 399/1-1 at Bielefeld and INTAS grant № 03-51-5018; the second author was supported by RFBF-DFG grant № 04-01-04000. 
The goal of this paper is to illustrate the possibilities of Stein's method for the investigation of Stieltjes transform of empirical spectral distribution function of random matrices and some of its applications. A similar approach based on the representation of type (7.5) and (7.6) in random matrix theory was used for the first time by Pastur for investigation of the Stieltjes transform of random matrices of the Wigner ensemble (see, for instance, [12], [7], [10]).

We state some general conditions of the convergence of the expected distribution function of random matrices to the semicircle law (of not necessarily independent entries) and give some applications of the main result. In Section 2 we derive a Stein-type equation for the semicircle law and provide on this basis some criteria of convergence to this law. We shall assume that $\mathbf{E} X_{j l}=0$ and $\sigma_{j l}^{2}:=\mathbf{E} X_{j l}^{2}$, for $1 \leqslant j \leqslant l \leqslant n$. Introduce, for any $j, l$, $1 \leqslant j \leqslant l \leqslant n$, the $\sigma$-algebras $\mathscr{F}(j l)=\sigma\left\{X_{k q}: 1 \leqslant k \leqslant q \leqslant n,(k, q) \neq(j, l)\right\}$ and $\mathscr{F}^{(j)}=\sigma\left\{X_{k q}: 1 \leqslant k \leqslant q \leqslant n, k \neq j\right.$ and $\left.q \neq j\right\}, 1 \leqslant j \leqslant n$. We introduce as well Lindeberg's ratio for random matrices, that is, for any $\tau>0$,

$$
L_{n}(\tau)=\frac{1}{n^{2}} \sum_{j=1}^{n} \sum_{l=1}^{n} \mathbf{E} X_{j l}^{2} I_{\left\{\left|X_{j l}\right|>\tau \sqrt{n}\right\}} .
$$

Furthermore, we use the notation $X_{j l}^{(\tau)}:=X_{j l} I_{\left\{\left|X_{j l}\right| \leqslant \tau \sqrt{n}\right\}}-\mathbf{E} X_{j l} I_{\left\{\left|X_{j l}\right| \leqslant \tau \sqrt{n}\right\}}$, $\left(\sigma_{j l}^{(\tau)}\right)^{2}=\mathbf{E}\left(X_{j l}^{(\tau)}\right)^{2}$.

Theorem 1.1. Assume that the random variables $X_{j l}, 1 \leqslant j \leqslant n$, $1 \leqslant l \leqslant n, n \geqslant 1$, satisfy the following conditions:

(a) for any $\tau>0$

$$
\begin{gathered}
\mathbf{E}\left\{X_{j l}^{(\tau)} \mid \mathscr{F}^{(j l)}\right\}=0 \\
\max _{1 \leqslant n<\infty} \max _{1 \leqslant j \leqslant n} \frac{1}{n} \sum_{l=1}^{n} \sigma_{j l}^{2} \leqslant C<\infty \\
\varepsilon_{n}^{(1)}:=\frac{1}{n^{2}} \sum_{j=1}^{n} \sum_{l=1}^{n} \mathbf{E}\left|\mathbf{E}\left\{\left(X_{j l}^{(\tau)}\right)^{2} \mid \mathscr{F}^{(j)}\right\}-\left(\sigma_{j l}^{(\tau)}\right)^{2}\right| \rightarrow 0
\end{gathered}
$$

(b) there exists $\sigma^{2}>0$ such that

$$
\begin{aligned}
\varepsilon_{n}^{(2)}: & =\frac{1}{n^{2}} \sum_{j=1}^{n} \sum_{l=1}^{n}\left|\sigma_{j l}^{2}-\sigma^{2}\right| \longrightarrow 0 \quad \text { as } n \rightarrow \infty, \\
\varepsilon_{n}^{(3)}:= & \frac{1}{n^{3}} \sum_{j=1}^{n} \sum_{1 \leqslant l \neq k \leqslant n} \mathbf{E} \mid \mathbf{E}\left\{\left(\left(X_{j k}^{(\tau)}\right)^{2}-\mathbf{E}\left(X_{j k}^{(\tau)}\right)^{2}\right)\right. \\
& \left.\times\left(\left(X_{j l}^{(\tau)}\right)^{2}-\mathbf{E}\left(X_{j l}^{(\tau)}\right)^{2}\right) \mid \mathscr{F}^{(j)}\right\} \mid \rightarrow 0 \quad \text { as } n \rightarrow \infty ;
\end{aligned}
$$

(c) for any fixed $\tau>0$

$$
L_{n}(\tau) \rightarrow 0 \quad \text { as } n \rightarrow \infty
$$


Then

$$
\Delta_{n}:=\sup _{x}\left|\mathbf{E} F_{n}(x)-G\left(x \sigma^{-1}\right)\right| \longrightarrow 0 \quad \text { as } n \rightarrow \infty .
$$

By $\|\mathbf{W}\|$ we denote the Frobenius norm of a matrix $\mathbf{W}:\|\mathbf{W}\|^{2}=$ $\sum_{j=1}^{n}\left|\lambda_{j}\right|^{2}=\sum_{j, k=1}^{n}|W(j, k)|^{2}$.

$\mathrm{R}$ e $\mathrm{m}$ a $\mathrm{r} \mathrm{k}$ 1.1. Note that condition (1.8) implies that

$$
\lim _{n \rightarrow \infty} n^{-1} \mathbf{E}\|\mathbf{W}\|^{2}=\sigma^{2}<\infty \text {. }
$$

Corollary 1.1. Let $X_{l j}, 1 \leqslant l \leqslant j<\infty$, be independent and $\mathbf{E} X_{l j}=0$, $\mathbf{E} X_{l j}^{2}=\sigma^{2}$. Assume that, for any fixed $\tau>0, L_{n}(\tau) \longrightarrow 0$ as $n \rightarrow \infty$. Then the expected spectral distribution function of matrix $\mathbf{W}$ converges to the distribution function of the semicircle law, that is,

$$
\Delta_{n}:=\sup _{x}\left|\mathbf{E} F_{n}(x)-G\left(x \sigma^{-1}\right)\right| \longrightarrow 0 \quad \text { as } n \rightarrow \infty .
$$

The investigation of the convergence of the spectral distribution functions of real symmetric random matrices with independent entries has a long history. For details see, for example, [1]. Convergence assuming Lindeberg's condition (Corollary 1.1) was proved in [13].

Another application of Theorem 1.1 is the distribution of spectra for the unitary invariant ensemble of real symmetric $n \times n$ matrices $\mathbf{W}_{n}=$ $\left(n^{-1 / 2} X_{l j}^{(n)}\right)$ induced by the uniform distribution on the sphere of the radius $\sqrt{N}$ in $\mathbf{R}^{N}$ with $N=n(n+1) / 2$, that is,

$$
\sum_{1 \leqslant l \leqslant j \leqslant n}\left(X_{l j}^{(n)}\right)^{2}=N .
$$

Rosenzweig in [14] defined this class of random matrices as a fixed-trace ensemble and proved the semicircle law using Wigner's method of moments. This class is described in [9, Chap. 19] as well.

Corollary 1.2. Let $X_{l j}^{(n)}, 1 \leqslant l \leqslant j \leqslant n$, for any $n \geqslant 1$, be distributed as above. Then

$$
\Delta_{n}:=\sup _{x}\left|\mathbf{E} F_{n}(x)-G(x)\right| \longrightarrow 0 \quad \text { as } n \rightarrow \infty .
$$

We may consider the ensemble of real symmetric $n \times n$ matrices determined by the uniform distribution in the ball of the radius $\sqrt{N}$ in $\mathbf{R}^{N}$ with $N=n(n+1) / 2$, that means that $\sum_{1 \leqslant l \leqslant j \leqslant n}\left(X_{l j}^{(n)}\right)^{2} \leqslant N$. This class of random matrices was introduced by Bronk in [2] as a bounded-trace ensemble. The eigenvalue density for the bounded-trace ensemble is identical to the density of zeros of Hermite-like polynomials. Using this fact, Bronk proved the semicircle law for such matrices. See also [9, Chap. 19].

Corollary 1.3. Let $X_{l j}^{(n)}, 1 \leqslant l \leqslant j \leqslant n$, be distributed uniformly in the ball of the radius $\sqrt{N}$ in $\mathbf{R}^{N}$ with $N=n(n+1) / 2$, for any $n \geqslant 1$. Then

$$
\Delta_{n} \rightarrow 0 \quad \text { as } n \rightarrow \infty \text {. }
$$




\section{Proofs of the Corollaries.}

$\mathrm{Pr}$ o of of $\mathrm{C}$ or o ll a r y 1.1. It is easy to check that all conditions of Theorem 1.1 hold.

$\mathrm{Pr}$ o of of $\mathrm{C}$ or oll a r y 1.2. To prove the corollary we have to examine the conditions of Theorem 1.1 only. Since all one-dimensional distributions are symmetric and the random variables $X_{l j}^{(n)}, 1 \leqslant l \leqslant j \leqslant n$, are exchangeable, conditions (1.8) and (1.6) hold with $C=\sigma^{2}=1$. It is easy to see that the conditional distribution of $X_{l j}$ given $\mathscr{F}^{(l j)}$ is symmetric. This yields condition (1.5). To check conditions (1.7) we note that, since $X_{l j}$ are exchangeable,

$$
\varepsilon_{n}^{(1)}:=\frac{1}{n^{2}} \sum_{1 \leqslant j \leqslant l \leqslant n} \mathbf{E}\left|\mathbf{E}\left\{X_{j l}^{2} \mid \mathscr{F}^{(j)}\right\}-\sigma^{2}\right|=\mathbf{E}\left|\mathbf{E}\left\{X_{11}^{2} \mid \mathscr{F}^{(1)}\right\}-1\right| .
$$

Furthermore, using equality (1.11), we get

$$
\mathbf{E}\left\{X_{11}^{2} \mid \mathscr{F}^{(1)}\right\}=N-\sum_{l=2}^{n} \mathbf{E}\left\{X_{1 l}^{2} \mid \mathscr{F}^{(1)}\right\}-\sum_{2 \leqslant l \leqslant j \leqslant n} X_{l j}^{2} .
$$

This implies that $\sum_{l=1}^{n} \mathbf{E}\left\{X_{1 l}^{2} \mid \mathscr{F}^{(1)}\right\}=\sum_{l=1}^{n} X_{1 l}^{2}$. Since $X_{1 l}, l=$ $1, \ldots, n$, are conditionally exchangeable given $\mathscr{F}^{(1)}$, we have $\mathbf{E}\left\{X_{1 l}^{2} \mid \mathscr{F}^{(1)}\right\}=$ $n^{-1} \sum_{l=1}^{n} X_{1 l}^{2}$.

Applying Cauchy's inequality, we get

$$
\mathbf{E}\left|\mathbf{E}\left\{X_{1 l}^{2} \mid \mathscr{F}^{(1)}\right\}-1\right| \leqslant \frac{1}{n}\left(\mathbf{E}\left|\sum_{l=1}^{n}\left(X_{1 l}^{2}-1\right)\right|^{2}\right)^{1 / 2} .
$$

Direct calculations show that, for $l \neq j, \mathbf{E}\left(X_{1 l}^{2}-1\right)\left(X_{1 j}^{2}-1\right)=2 / N$. The last relation yields $\mathbf{E}\left|\mathbf{E}\left\{X_{1 l}^{2} \mid \mathscr{F}^{(1)}\right\}-1\right| \leqslant C / \sqrt{n}$. Checking of condition (1.9) is similar. The Lindeberg condition (1.10) follows from the symmetry of the distribution and the boundedness of the random variables. This concludes the proof.

$\mathrm{Pr}$ o of of $\mathrm{C}$ or o lla r y 1.3. We have to check the conditions of Theorem 1.1. Conditions (1.5), (1.8), and (1.10) hold by the same reason as in Corollary 1.2. We now check condition (1.7). Since $X_{11}, \ldots, X_{1 n}$ have a conditionally exchangeable distribution given $\mathscr{F}^{(1)}$, we get

This implies that

$$
\mathbf{E}\left\{X_{1 l}^{2} \mid \mathscr{F}^{(1)}\right\}=\frac{1}{n} \mathbf{E}\left\{\sum_{l=1}^{n} X_{1 l}^{2} \mid \mathscr{F}^{(1)}\right\} .
$$

$$
\mathbf{E}\left|\mathbf{E}\left\{X_{11}^{2} \mid \mathscr{F}^{(1)}\right\}-\mathbf{E} X_{11}^{2}\right| \leqslant \mathbf{E}\left|\frac{1}{n} \sum_{l=1}^{n}\left(X_{1 l}^{2}-\mathbf{E} X_{11}^{2}\right)\right| .
$$

A simple calculation shows that

$$
\mathbf{E} X_{11}^{2} X_{12}^{2}-\mathbf{E} X_{11}^{2} \mathbf{E} X_{12}^{2}=-\frac{2 N^{2}}{(N+4)(N+2)^{2}} \text {. }
$$

This implies that $\mathbf{E}\left|\mathbf{E}\left\{X_{11}^{2} \mid \mathscr{F}^{(1)}\right\}-\mathbf{E} X_{11}^{2}\right| \leqslant C / \sqrt{n}$, which completes the proof. 
3. Stein's equation for the semicircle law. We start from a simple characterization of the semicircle law. Let $\mathbb{C}(\mathbf{R})$ (respectively, $\mathbb{C}^{\mathbf{l}}(B)$, $B \subset \mathbf{R}$ ) denote the class of continuous functions on $\mathbf{R}$ (respectively, the class of differentiable functions on $B$ with bounded first derivatives on all compact subsets of $B$ ). Introduce a class of bounded functions without discontinuity of second order via

$$
\begin{aligned}
& \mathbb{C}_{\{-2,2\}}^{1}=\left\{f: \mathbf{R} \rightarrow \mathbf{R}: f \in \mathbb{C}^{1}(\mathbf{R} \backslash\{-2,2\}) ;\right. \\
&\left.\varlimsup_{|y| \rightarrow \infty}|y \cdot f(y)|<\infty ; \limsup _{y \rightarrow \pm 2}\left|4-y^{2} \| f^{\prime}(y)\right|<C\right\} .
\end{aligned}
$$

At first we prove the following result.

Lemma 3.1. Assume that a bounded function $\varphi(x)$ without discontinuity of second order satisfies the following conditions:

$\varphi(x)$ is continuous at the points $x= \pm 2, \quad \int_{-2}^{2} \varphi(u) \sqrt{4-u^{2}} d u=0$.

Then there exists a function $f \in \mathbb{C}_{\{-2,2\}}^{1}$ such that, for any $x \neq \pm 2$,

$$
\left(4-x^{2}\right) f^{\prime}(x)-3 x f(x)=\varphi(x) \text {. }
$$

If $\varphi( \pm 2)=0$, then there exists a continuous solution of the equation (3.1).

P r o o f. We define the following function $f(x)$. Let $x<-2$. Then

$$
f(x):=-\exp \left\{-\int_{-2}^{x} \frac{3 u d u}{u^{2}-4}\right\} \int_{-2}^{x} \exp \left\{\int_{-2}^{u} \frac{3 v d v}{v^{2}-4}\right\} \frac{\varphi(u) d u}{u^{2}-4} .
$$

After integration we get

$$
f(x):=-\frac{1}{\left(x^{2}-4\right)^{3 / 2}} \int_{-2}^{x} \varphi(u) \sqrt{u^{2}-4} d u .
$$

Analogously, we define for $x>2$

$$
f(x):=-\frac{1}{\left(x^{2}-4\right)^{3 / 2}} \int_{2}^{x} \varphi(u) \sqrt{u^{2}-4} d u .
$$

For $x \in(-2,2)$, we have

$$
f(x):=\frac{1}{\left|x^{2}-4\right|^{3 / 2}} \int_{-2}^{x} \varphi(u) \sqrt{4-u^{2}} d u .
$$

It is straightforward to show that the above function $f(x)$ satisfies the equation (3.1). We calculate now

$$
a:=\lim _{x \searrow 2} f(x), \quad b:=\lim _{x \nearrow-2} f(x), \quad c:=\lim _{x \nearrow 2} f(x), \quad d:=\lim _{x \backslash-2} f(x) .
$$

According to de l'Hôpital's rule, we get $a=-c=-\frac{1}{6} \varphi(2), b=-d=$ $\frac{1}{6} \varphi(-2)$. The function $f(x)$ has jumps at the point $x= \pm 2$ if $\varphi( \pm 2) \neq 0$ only. The representation (3.2)-(3.4) together imply that $\lim _{\sup _{y \rightarrow \infty}}|y f(y)|<\infty$ and lim $\sup _{y \rightarrow \pm 2}\left|\left(y^{2}-4\right) f^{\prime}(y)\right|<\infty$. This concludes the proof of the lemma. 
Proposition 3.1. The random variable $\xi$ has distribution function $G(x)$ if and only if the following equality holds, for any function $f \in \mathbb{C}_{\{-2,2\}}^{1}$ :

$$
\mathbf{E}\left(4-\xi^{2}\right) f^{\prime}(\xi)-3 \mathbf{E} \xi f(\xi)=0 .
$$

$\mathrm{P}$ r o of. At first we proof the necessity. Assume that $\xi$ has distribution function $G(x)$. By definition of the class $\mathbb{C}_{\{-2,2\}}^{1}$, the function $\left(4-y^{2}\right) g(y) f^{\prime}(y)=(2 \pi)^{-1}\left(4-y^{2}\right)^{3 / 2} f^{\prime}(y) I_{[-2,2]}(y)$ is continuous and $\mathbf{E}\left|\left(4-\xi^{2}\right) f^{\prime}(\xi)\right|<\infty$. Integrating by parts, we get

$$
\begin{aligned}
\mathbf{E}\left(4-\xi^{2}\right) f^{\prime}(\xi) & =\int_{-2}^{2}\left(4-y^{2}\right) g(y) f^{\prime}(y) d y \\
& =-\int_{-2}^{2} f(y)\left[-2 y g(y)+\left(4-y^{2}\right) g^{\prime}(y)\right] d y .
\end{aligned}
$$

Using definition (1.3), it is easy to check that, for $y \in[-2,2]$,

$$
\left(4-y^{2}\right) g^{\prime}(y)=-y g(y) \text {. }
$$

Substituting (3.7) in (3.6) we obtain (3.5). Now we prove the sufficiency of condition (3.5). According to Lemma 3.1, for any fixed number $x$, there exists a function $f^{(x)}(y) \in \mathbb{C}_{\{-2,2\}}^{1}$ such that the following equation holds:

$$
\left(4-y^{2}\right)\left(f^{(x)}(y)\right)_{y}^{\prime}-3 y f^{(x)}(y)=h_{x}(y)-G(x),
$$

where $h_{x}(y)$ is the indicator function of the interval $(-\infty, x)$.

Evaluating the expectation of $\left(4-\xi^{2}\right)\left(f^{(x)}(y)\right)_{x}^{\prime}(\xi)-3 \xi f^{(x)}(\xi)$, we get that, for any $x, \mathbf{P}\{\xi \leqslant x\}=G(x)$, which proves the proposition.

3.1. A Stein equation for random matrices. Let $W$ denote a symmetric random matrix with eigenvalues $\lambda_{1} \leqslant \cdots \leqslant \lambda_{n}$. If $\mathbf{W}=\mathbf{U}^{-1} \boldsymbol{\Lambda} \mathbf{U}$, where $\boldsymbol{U}$ is an orthogonal matrix and $\boldsymbol{\Lambda}$ is a diagonal matrix, one defines $f(\mathbf{W})=\mathbf{U}^{-1} f(\boldsymbol{\Lambda}) \mathbf{U}$, where $f(\boldsymbol{\Lambda})=\operatorname{diag}\left(f\left(\lambda_{1}\right), \ldots, f\left(\lambda_{n}\right)\right)$. We denote by $\mathbf{I}_{n}$ the identity matrix of order $n \times n$.

We can now formulate the convergence to the semicircle law for the spectral distribution function of random matrices.

Theorem 3.1. Let $\mathbf{W}_{n}$ denote a sequence of random matrices of order $n \times n$ such that, for any function $f \in \mathbb{C}_{\{-2,2\}}^{1}$,

$$
n^{-1} \mathbf{E} \operatorname{Tr}\left(4 \mathbf{I}_{n}-\mathbf{W}_{n}^{2}\right) f^{\prime}\left(\mathbf{W}_{n}\right)-3 n^{-1} \mathbf{E} \operatorname{Tr} \mathbf{W}_{n} f\left(\mathbf{W}_{n}\right) \longrightarrow 0 \quad \text { as } n \rightarrow \infty \text {. }
$$

Then

$$
\Delta_{n}:=\sup _{x}\left|\mathbf{E} F_{n}(x)-G(x)\right| \longrightarrow 0 \quad \text { as } n \rightarrow \infty .
$$

P r o o f. For any function $f \in \mathbb{C}_{\{-2,2\}}^{1}$, introduce the operator

$$
\mathscr{L}_{n}(f):=n^{-1} \mathbf{E} \operatorname{Tr}\left(4 \mathbf{I}_{n}-\mathbf{W}_{n}^{2}\right) f^{\prime}\left(\mathbf{W}_{n}\right)-3 n^{-1} \mathbf{E} \operatorname{Tr} \mathbf{W}_{n} f\left(\mathbf{W}_{n}\right) .
$$

There exists, for any $x$, a solution of equation (3.8) $f^{(x)} \in \mathbb{C}_{\{-2,2\}}^{1}$ such that

$$
\mathscr{L}_{n}\left(f^{(x)}\right)=G_{n}(x)-G(x),
$$


where $G_{n}(x):=\mathbf{E} F_{n}(x)$. To prove (3.10) we introduce a random variable $J$, which is uniformly distributed on $\{1, \ldots, n\}$ and which is independent on $X_{l j}$, $1 \leqslant l \leqslant j \leqslant n$. Note that

$$
\mathbf{P}\left\{\lambda_{J} \leqslant x\right\}=\frac{1}{n} \sum_{j=1}^{n} \mathbf{P}\left\{\lambda_{j} \leqslant x\right\}=\frac{1}{n} \sum_{j=1}^{n} \mathbf{E} I_{\left\{\lambda_{j} \leqslant x\right\}}=\mathbf{E} F_{n}(x) .
$$

Consider equality (3.8) for $y=\lambda_{J}$ :

$$
\left(4-\lambda_{J}^{2}\right)\left(f^{(x)}\left(\lambda_{J}\right)\right)_{y}^{\prime}-3 \lambda_{J} f^{(x)}\left(\lambda_{J}\right)=h_{x}\left(\lambda_{J}\right)-G(x) .
$$

Taking expectation of the both sides of (3.11), we obtain (3.10). Relations (3.9), (3.10) together complete the proof.

For any $L>0$, consider the class of function $\mathbb{C}_{\{-2,2, L\}}^{1}:=\left\{f \in \mathbb{C}_{\{-2,2\}}^{1}\right.$ : $f(y)=0$, for $y \notin[-L, L]\}$.

Corollary 3.1. Assume that, for some positive constant $C$,

$$
\limsup _{n \rightarrow \infty} \frac{1}{n} \mathbf{E}\left\|\mathbf{W}_{n}\right\|^{2} \leqslant C .
$$

Assume that, for any $L>0$ and for any $f \in \mathbb{C}_{\{-2,2, L\}}^{1}, \mathscr{L}_{n}(f) \rightarrow 0$ as $n \rightarrow \infty$. Then

$$
\Delta_{n}:=\sup _{x}\left|\mathbf{E} F_{n}(x)-G(x)\right| \longrightarrow 0 \quad \text { as } n \rightarrow \infty .
$$

P r o of. Let $L>0$ be fixed. Consider the function $\tilde{f}_{x}(y)=$ $f^{(x)}(y) I_{[-L, L]}(y)$. According to (3.8) we have

$$
\begin{aligned}
\mathscr{L}_{n}\left(\tilde{f}_{x}\right) & =\frac{1}{n} \sum_{j=1}^{n} \mathbf{E}\left(I_{\left\{\lambda_{j} \leqslant x\right\}}-G(x)\right) I_{\left\{\left|\lambda_{j}\right| \leqslant L\right\}} \\
& =G_{n}(x)-G(x)-\frac{1}{n} \sum_{j=1}^{n} \mathbf{E}\left(I_{\left\{\lambda_{j} \leqslant x\right\}}-G(x)\right) I_{\left\{\left|\lambda_{j}\right| \geqslant L\right\}} .
\end{aligned}
$$

This equality implies that

$$
\begin{aligned}
\limsup _{n \rightarrow \infty}\left|G_{n}(x)-G(x)\right| & \leqslant \limsup _{n \rightarrow \infty} \frac{1}{n} \sum_{j=1}^{n} \mathbf{E} I_{\left\{\left|\lambda_{j}\right| \geqslant L\right\}} \leqslant \frac{1}{L^{2}} \limsup _{n \rightarrow \infty} \frac{1}{n} \sum_{j=1}^{n} \mathbf{E}\left|\lambda_{j}\right|^{2} \\
& \leqslant \frac{1}{L^{2}} \limsup _{n \rightarrow \infty} \frac{1}{n} \mathbf{E}\left\|\mathbf{W}_{n}\right\|^{2} .
\end{aligned}
$$

Since the last limit on the right-hand side of (3.12) is finite and $L$ is arbitrary, we get $\lim \sup _{n \rightarrow \infty}\left|G_{n}(x)-G(x)\right|=0$. This concludes the proof.

Let $\mathbf{W}_{n}=\mathbf{U}_{n}^{T} \boldsymbol{\Lambda}_{n} \mathbf{U}_{n}$, where $\boldsymbol{\Lambda}_{n}=\operatorname{diag}\left(\lambda_{1}, \ldots, \lambda_{n}\right)$. We introduce the truncated matrix $\mathbf{W}_{n}^{(L)}:=\mathbf{U}_{n}^{T} \Lambda_{n}^{(L)} \mathbf{U}_{n}$, where $\Lambda_{n}^{(L)}=\operatorname{diag}\left(\lambda_{1}^{(L)}, \ldots, \lambda_{n}^{(L)}\right)$ and $\lambda_{j}^{(L)}=\lambda_{j} I_{\left\{\left|\lambda_{j}\right| \leqslant L\right\}}$. The expected empirical spectral distribution function of the matrix $\mathbf{W}_{n}^{(L)}$ is defined by

$$
\mathbf{E} F_{n}^{(L)}(x):=\frac{1}{n} \sum_{j=1}^{n} \mathbf{E} I_{\left\{\lambda_{j}^{(L)} \leqslant x\right\}} .
$$


It is easy to see that

$$
\left|\mathbf{E} F_{n}(x)-\mathbf{E} F_{n}^{(L)}(x)\right| \leqslant \frac{1}{n} \sum_{j=1}^{n} \mathbf{E} I_{\left\{\left|\lambda_{j}\right| \geqslant L\right\}} \leqslant \frac{1}{L^{2}} \frac{1}{n} \mathbf{E}\left\|\mathbf{W}_{n}\right\|^{2} .
$$

4. The Stieltjes transform. Introduce the Stieltjes transform of a random variable $\xi$ with distribution function $F(x)$ : for any $z=u+i v, v \neq 0$,

$$
T(z)=\mathbf{E} \frac{1}{\xi-z}=\int_{-\infty}^{\infty} \frac{1}{x-z} d F(x) .
$$

In random matrix theory the Stieltjes transform of random variables was used for the first time by Marchenko and Pastur [8].

Note that $T(z)$ is analytic for nonreal $z$ and satisfies the conditions

$$
\operatorname{Im} T \cdot \operatorname{Im} z>0, \quad \operatorname{Im} z \neq 0, \quad \sup _{v \geqslant 1} v|T(i v)|=1 .
$$

It can be shown that for any continuous function $\varphi(\lambda)$ with compact support

$$
\int_{-\infty}^{\infty} \varphi(\lambda) d F(\lambda)=\lim _{v \rightarrow 0} \frac{1}{\pi} \int_{-\infty}^{\infty} \varphi(\lambda) \operatorname{Im} T(\lambda+i v) d \lambda
$$

Furthermore, the one-to-one correspondence between distribution functions and their Stieltjes transforms is continuous with respect to the weak convergence of distribution functions as well as with respect to the uniform convergence on compacts sets in $\mathbf{C} \backslash \mathbf{R}$.

4.1. The Stieltjes transform of a semicircle law. Introduce the function $f_{z}(x)=(x-z)^{-1}$, for any nonreal $z$. Note that $\left(f_{z}(x)\right)_{x}^{\prime}=$ $-\left(f_{z}(x)\right)_{z}^{\prime}=-1 /(x-z)^{2}$. Denote by $S(z)$ the Stieltjes transform of the semicircle law. By definition of the Stieltjes transform, we have

$$
S(z)=\int_{-\infty}^{\infty} \frac{1}{x-z} d G(x)=\mathbf{E} f_{z}(\xi), \quad S^{\prime}(z)=-\mathbf{E}\left(f_{z}(\xi)\right)_{x}^{\prime}=\mathbf{E} \frac{1}{(\xi-z)^{2}}
$$

where $\xi$ is a random variable with the distribution function $G(x)$. Applying now equation (3.5), we obtain

$$
\mathbf{E} \frac{4-\xi^{2}}{(\xi-z)^{2}}+3 \mathbf{E} \frac{\xi}{\xi-z}=0
$$

Combining (4.2), (4.1), and the obvious relations

$$
\frac{4-\xi^{2}}{(\xi-z)^{2}}=\frac{4-z^{2}}{(\xi-z)^{2}}-1-\frac{2 z}{\xi-z}
$$

and

$$
\frac{\xi}{\xi-z}=1+\frac{z}{\xi-z}
$$

we obtain a differential equation for the Stieltjes transform of the semicircle law

$$
\left(z^{2}-4\right) S^{\prime}(z)-z S(z)-2=0
$$


Solving this differential equation we obtain, for $z$ and $z_{0}$ such that $\operatorname{Im} z$. $\operatorname{Im} z_{0}>0$,

$$
S(z)=\frac{\left(z^{2}-4\right)^{1 / 2}}{\left(z_{0}^{2}-4\right)^{1 / 2}}\left(S\left(z_{0}\right)+2\left(z_{0}^{2}-4\right)^{1 / 2} \int_{z_{0}}^{z} \frac{d u}{\left(u^{2}-4\right)^{3 / 2}}\right),
$$

where $\sqrt{u^{2}-4}=\sqrt{u-2} \sqrt{u+2}$ (principal argument). Since $S(\bar{z})=\overline{S(z)}$, it is sufficient to calculate this integral for $\operatorname{Im} z>0$ only. Putting $u=\zeta+\zeta^{-1}$ and $\zeta(u)=\frac{1}{2}\left(u+\sqrt{u^{2}-4}\right)$, we obtain

$$
\int_{z_{0}}^{z} \frac{d u}{\left(u^{2}-4\right)^{3 / 2}}=\int_{\zeta\left(z_{0}\right)}^{\zeta(z)} \frac{\zeta d \zeta}{\left(\zeta^{2}-1\right)^{2}}=\frac{1}{2}\left[\frac{1}{\zeta^{2}\left(z_{0}\right)-1}-\frac{1}{\zeta^{2}(z)-1}\right] .
$$

It is easy to check that

$$
\left(\zeta^{2}(u)-1\right)^{-1}=\frac{u-\sqrt{u^{2}-4}}{2 \sqrt{u^{2}-4}}
$$

Substituting (4.7) in (4.6), we obtain

$$
S(z)=\frac{\left(z^{2}-4\right)^{1 / 2}}{\left(z_{0}^{2}-4\right)^{1 / 2}}\left(S\left(z_{0}\right)+\frac{1}{2}\left(z_{0}-\sqrt{z_{0}^{2}-4}\right)\right)-\frac{1}{2}\left(z-\sqrt{z^{2}-4}\right) .
$$

Since $\operatorname{Im} \sqrt{z_{0}^{2}-4}>0$,

$$
\frac{1}{2}\left|z_{0}-\sqrt{z_{0}^{2}-4}\right|=\frac{2}{\left|z_{0}+\sqrt{z_{0}^{2}-4}\right|} \leqslant \frac{2}{\operatorname{Im}\left\{z_{0}+\sqrt{z_{0}^{2}-4}\right\}} \leqslant 2 v_{0}^{-1} .
$$

According to (4.1), for $z_{0}=u_{0}+i v_{0}$, we have $\left|S\left(z_{0}\right)\right| \leqslant v_{0}^{-1}$. Passing to the limit in equality (4.8) in $z_{0}=u_{0}+i v_{0}$ as $v_{0} \rightarrow \infty$, we get the following formula for the Stieltjes transform of the semicircle law, for all $z$ such that $\operatorname{Im} z>0: S(z)=-\frac{1}{2}\left(z-\sqrt{z^{2}-4}\right)$. This formula implies a simple algebraic equation for the Stieltjes transform of the semicircle law, namely

$$
S^{2}(z)+z S(z)+1=0 .
$$

\section{The Stieltjes transform of the spectral distribution function} of a random matrix. In what follows we shall omit the subindex $n$ in the notation of matrices. Recall that the spectral distribution function of a random symmetric matrix $\mathbf{W}$ is defined as

$$
F_{n}(x)=\frac{1}{n} \sum_{j=1}^{n} I_{\left\{\lambda_{j} \leqslant x\right\}},
$$

where $\lambda_{1}, \ldots, \lambda_{n}$ are the eigenvalues of $\mathbf{W}$.

We introduce the resolvent matrix for a symmetric matrix $\mathbf{W}$ : for any nonreal $z$,

$$
\mathbf{R}(z)=(\mathbf{W}-z \mathbf{I})^{-1},
$$

where I denotes the identity matrix of order $n \times n$. Introduce the Stieltjes transform of the spectral distribution function as

$$
M_{n}(z):=\int_{-\infty}^{\infty} \frac{1}{x-z} d F_{n}(x)=\frac{1}{n} \sum_{j=1}^{n} \frac{1}{\lambda_{j}-z}=\frac{1}{n} \operatorname{Tr} \mathbf{R}(z) .
$$


We shall consider also the Stieltjes transform of the expected spectral distribution function

$$
S_{n}(z):=\mathbf{E} M_{n}(z)=\int_{-\infty}^{\infty} \frac{1}{x-z} d \mathbf{E} F_{n}(x)=\frac{1}{n} \mathbf{E} \operatorname{Tr} \mathbf{R}(z) .
$$

5.1. Estimation of the variance of the Stieltjes transforms of spectral distribution functions of random matrices with dependent entries. Let $z=u+i v$. Let $\mathbf{W}_{j}$ denote the submatrix of $\mathbf{W}$ which is obtained from $\mathbf{W}$ by elimination of $j$ th column and $j$ th row. Put $\mathbf{R}_{j}=$ $\left(\mathbf{W}_{j}-z \mathbf{I}_{n-1}\right)$. We are interested in a bound for the quantity

$$
A:=n^{-2} \mathbf{E}|\operatorname{Tr} \mathbf{R}-\mathbf{E} \operatorname{Tr} \mathbf{R}|^{2} .
$$

We start with the following representation:

$$
R(j, j)=-\frac{1}{z+s_{n}(z)}\left(1-\eta_{j} R(j, j)\right)
$$

where $\eta_{j}=\eta_{j}^{(1)}+\eta_{j}^{(2)}+\eta_{j}^{(3)}+\eta_{j}^{(4)}$, with

$$
\begin{aligned}
\eta_{j}^{(1)} & :=\frac{1}{\sqrt{n}} X_{j j}, \quad \eta_{j}^{(2)}:=-\frac{1}{n}\left(\mathbf{a}_{j}^{T} \mathbf{R}_{j} \mathbf{a}_{j}-\sigma^{2} \operatorname{Tr} \mathbf{R}\right), \\
\eta_{j}^{(3)} & :=\sigma^{2} \frac{1}{n}\left(\operatorname{Tr} \mathbf{R}-\operatorname{Tr} \mathbf{R}_{j}\right), \quad \eta_{j}^{(4)}:=-\sigma^{2} \frac{1}{n}(\operatorname{Tr} \mathbf{R}-\mathbf{E} \operatorname{Tr} \mathbf{R}) .
\end{aligned}
$$

At first we prove the following lemma.

Lemma 5.1. Assuming the conditions of Theorem 1.1 we have

$$
A=\frac{1}{n} \sum_{j=1}^{n} \mathbf{E}\left|\eta_{j}^{(4)}\right|^{2} \longrightarrow 0 \quad \text { as } n \rightarrow \infty
$$

$\mathrm{P}$ r o o f. Introduce the matrix with truncated entries,

$$
\mathbf{W}^{(c)}=\left(n^{-1 / 2}\left(X_{j k}^{(\tau)}-\mathbf{E} X_{j k}^{(\tau)}\right)\right)_{j, k=1}^{n},
$$

where $X_{j k}^{(\tau)}:=X_{j k} I_{\left\{\left|X_{j k}\right| \leqslant \tau \sqrt{n}\right\}}$. Let $\mathbf{W}^{(t)}=\mathbf{W}-\mathbf{W}^{(c)}$ and $\mathbf{R}^{(c)}=\left(\mathbf{W}^{(c)}-\right.$ $\left.z \mathbf{I}_{n}\right)^{-1}$ and $\mathbf{R}^{(t)}=\left(\mathbf{W}^{(t)}-z \mathbf{I}_{n}\right)^{-1}$. Note that $\mathbf{E} n^{-1} \operatorname{Tr}\left(\mathbf{W}^{(t)}\right)^{2} \leqslant \mathscr{L}_{n}(\tau)$. By resolvent equality we have

$$
\mathbf{R}=\mathbf{R}^{(c)}-z \mathbf{R}^{(c)} \mathbf{W}^{(t)} \mathbf{R} .
$$

This implies

$$
\begin{aligned}
& \mathbf{E}\left|n^{-1}(\operatorname{Tr} \mathbf{R}-\mathbf{E} \operatorname{Tr} \mathbf{R})\right|^{2} \leqslant \mathbf{E}\left|n^{-1}\left(\operatorname{Tr} \mathbf{R}^{(c)}-\mathbf{E} \operatorname{Tr} \mathbf{R}^{(c)}\right)\right|^{2} \\
& \quad+C v^{-4} n^{-1} \mathbf{E} \operatorname{Tr}\left(\mathbf{W}^{(t)}\right)^{2} \leqslant \mathbf{E}\left|n^{-1}\left(\operatorname{Tr} \mathbf{R}^{(c)}-\mathbf{E} \operatorname{Tr} \mathbf{R}^{(c)}\right)\right|^{2}+C v^{-4} \mathscr{L}_{n}(\tau) .
\end{aligned}
$$

Let $A^{(c)}:=\mathbf{E}\left|n^{-1}\left(\operatorname{Tr} \mathbf{R}^{(c)}-\mathbf{E} \operatorname{Tr} \mathbf{R}^{(c)}\right)\right|^{2}$. At first we assume that $v \geqslant 2$. Using representation (5.1), we get

$$
\begin{aligned}
A^{(c)} & \leqslant \frac{1}{\left|z+S_{n}(z)\right|^{2}} \frac{1}{n} \sum_{j=1}^{n} \mathbf{E}\left|\eta_{j}^{(c)} R^{(c)}(j, j)-\mathbf{E} \eta_{j}^{(c)} R^{(c)}(j, j)\right|^{2} \leqslant \frac{2}{v^{4}} \sum_{j=1}^{n} \mathbf{E}\left|\eta_{j}^{(c)}\right|^{2} \\
& \leqslant \frac{2}{v^{4}} A^{(c)}+\frac{2}{v^{4}} \sum_{j=1}^{n} \mathbf{E}\left|\eta_{j}^{(t, 1)}\right|^{2}+\frac{2}{v^{4}} \sum_{j=1}^{n} \mathbf{E}\left|\eta_{j}^{(t, 2)}\right|^{2}+\frac{2}{v^{4}} \sum_{j=1}^{n} \mathbf{E}\left|\eta_{j}^{(t, 3)}\right|^{2}
\end{aligned}
$$


where $\eta_{j}^{(t, 1)}=n^{-1 / 2} X_{j j}^{(c)}$,

$\eta_{j}^{(t, 2)}=-n^{-1}\left(\left(\mathbf{a}_{j}^{(c)}\right)^{T} \mathbf{R}_{j}^{(c)} \mathbf{a}_{j}^{(c)}-\sigma^{2} \operatorname{Tr} \mathbf{R}_{j}^{(c)}\right), \quad \eta_{j}^{(t, 3)}=\sigma^{2} n^{-1}\left(\operatorname{Tr} \mathbf{R}^{(c)}-\operatorname{Tr} \mathbf{R}_{j}^{(c)}\right)$.

It is easy to see that $\mathbf{E}\left|\eta_{j}^{(t, 1)}\right|^{2} \leqslant \sigma^{2} / n \rightarrow 0$ as $n \rightarrow \infty$.

To bound $\varkappa_{j}=\operatorname{Tr} \mathbf{R}-\operatorname{Tr} \mathbf{R}_{j}$ we need some auxiliary lemma. Let $\mathbf{A}$ be an $n \times n$ symmetric matrix, and let $\mathbf{A}^{(k)}$ denote a principal submatrix, obtained from $\mathbf{A}$ by deleting $k$-th row and column.

Lemma 5.2. The following bound holds: for $z=u+i v, v>0$,

$$
\left|\operatorname{Tr}\left(\mathbf{A}-z \mathbf{I}_{n}\right)^{-1}-\operatorname{Tr}\left(\mathbf{A}^{(k)}-z \mathbf{I}_{n-1}\right)^{-1}\right| \leqslant v^{-1} \text {. }
$$

P r o o f. Applying a Schur complement formula (see [6, Chap. 08, p. 21]) with $\mathbf{a}_{k}^{T}=\left(X_{k 1}, \ldots, X_{k, k-1}, X_{k, k+1}, \ldots, X_{k n}\right)$, we get

$$
\operatorname{det}\left(\mathbf{A}-z \mathbf{I}_{n}\right)=\left(n^{-1 / 2} X_{k k}-z-n^{-1} \mathbf{a}_{k}^{T}\left(\mathbf{A}^{(k)}-z \mathbf{I}_{n-1}\right)^{-1} \mathbf{a}_{k}\right) \operatorname{det}\left(\mathbf{A}^{(k)}-z \mathbf{I}_{n-1}\right) .
$$

Taking the logarithm of both sides of this equation and taking derivatives we obtain

$$
\operatorname{Tr}\left(\mathbf{A}-z \mathbf{I}_{n}\right)^{-1}-\operatorname{Tr}\left(\mathbf{A}^{(k)}-z \mathbf{I}_{n-1}\right)^{-1}=\frac{1+\mathbf{a}_{k}^{T}\left(\mathbf{A}^{(k)}-z \mathbf{I}_{n-1}\right)^{-2} \mathbf{a}_{k}}{a_{k k}-z-\mathbf{a}_{k}^{T}\left(\mathbf{A}^{(k)}-z \mathbf{I}_{n-1}\right)^{-1} \mathbf{a}_{k}}
$$

Let $\mathbf{T}$ be an orthogonal transformation which transforms $\mathbf{A}$ into diagonal form. Denote by $\mu_{1} \leqslant \cdots \leqslant \mu_{n-1}$ the eigenvalues of $\mathbf{A}_{k}$ and let $\left(y_{1}, \ldots, y_{n-1}\right)=\mathbf{a}_{k}^{T} \mathbf{T}^{T}$. Then

$$
\begin{aligned}
& \left|1+\mathbf{a}_{k}^{T}\left(\mathbf{A}^{(k)}-z \mathbf{I}_{n-1}\right)^{-2} \mathbf{a}_{k}\right|=\left|1+\sum_{l=1}^{n-1} y_{l}^{2}\left(\mu_{l}-z\right)^{-2}\right| \\
& \leqslant 1+\sum_{l=1}^{n-1} y_{l}^{2}\left(\left(\mu_{l}-u\right)^{2}+v^{2}\right)^{-1} \leqslant 1+\mathbf{a}_{k}^{T}\left(\left(\mathbf{A}^{(k)}-u \mathbf{I}_{n-1}\right)^{2}+v^{2} \mathbf{I}_{n-1}\right)^{-1} \mathbf{a}_{k} .
\end{aligned}
$$

Since for any commuting matrices $\mathbf{A}, \mathbf{B}$ such that $\mathbf{A}^{2}+\mathbf{B}^{2}$ is nondegenerate

$$
(\mathbf{A}+i \mathbf{B})^{-1}=(\mathbf{A}-i \mathbf{B})\left(\mathbf{A}^{2}+\mathbf{B}^{2}\right)^{-1},
$$

we can directly verify that

$$
\begin{aligned}
& \operatorname{Im}\left(a_{k k}-z-\mathbf{a}_{k}^{T}\left(\mathbf{A}^{(k)}-z \mathbf{I}_{n-1}\right)^{-1} \mathbf{a}_{k}\right) \\
& \quad=-v\left(1+\mathbf{a}_{k}^{T}\left(\left(\mathbf{A}^{(k)}-u \mathbf{I}_{n-1}\right)^{2}+v^{2} \mathbf{I}_{n-1}\right)^{-1} \mathbf{a}_{k}\right) .
\end{aligned}
$$

The last two relations yield the result.

Applying Lemma 5.2 with $\mathbf{A}=\mathbf{W}$ and $\mathbf{A}^{(k)}=\mathbf{W}^{(k)}$, we get

$$
\left|\varkappa_{k}\right| \leqslant v^{-1} \text {. }
$$

Similarly, we have $\mathbf{E}\left|\eta_{j}^{(t, 3)}\right|^{2} \leqslant n^{-2} v^{-2} \rightarrow 0$ as $n \rightarrow \infty$.

Now we prove that

$$
\mathrm{E}\left|\eta^{(j, 2)}\right|^{2} \longrightarrow 0 \quad \text { as } n \rightarrow \infty .
$$


We have

$$
\begin{aligned}
\mathbf{E}\left|\eta^{(j, 2)}\right|^{2}= & \frac{1}{n^{2}} \sum_{k=1}^{n} \mathbf{E E}\left\{\left(\left(X_{j k}^{(c)}\right)^{2}-\sigma^{2}\right)^{2} \mid \mathscr{F}^{(j)}\right\}\left|R_{j}(k, k)\right|^{2} \\
& +\frac{1}{n^{2}} \sum_{k \neq l} \mathbf{E E}\left\{\left(\left(X_{j k}^{(c)}\right)^{2}-\sigma^{2}\right)\left(\left(X_{j l}^{(c)}\right)^{2}-\sigma^{2}\right) \mid \mathscr{F}^{(j)}\right\} \\
& \times\left|R_{j}(k, k)\right|\left|R_{j}(l, l)\right| \\
& +\frac{1}{n^{2}} \sum_{k \neq l} \mathbf{E E}\left\{\left(X_{j k}^{(c)}\right)^{2}\left(X_{j l}^{(c)}\right)^{2} \mid \mathscr{F}^{(j)}\right\}\left|R_{j}(k, l)\right|^{2} \\
= & B_{1}^{(j)}+B_{2}^{(j)}+B_{3}^{(j)}+B_{4}^{(j)} .
\end{aligned}
$$

For $B_{1}$ the bound

$$
\frac{1}{n} \sum_{j=1}^{n} B_{1}^{(j)} \leqslant \tau^{2} \frac{1}{n^{2} v^{2}} \sum_{l, j=1}^{n} \mathbf{E} X_{j l}^{2} \leqslant C \tau^{2}
$$

holds. Analogously,

$$
\frac{1}{n} \sum_{j=1}^{n} B_{3}^{(j)} \leqslant \tau^{2} \frac{1}{n^{2} v^{2}} \sum_{j=1}^{n} \sum_{k \neq l} \mathrm{E}\left(X_{j k}^{(c)}\right)^{2}\left|R_{j}(k, l)\right|^{2} \leqslant \tau^{2} \frac{1}{n v^{2}} \sum_{j=1}^{n} \sum_{k=1}^{n} \mathrm{E} X_{j k}^{2} \leqslant C \tau^{2} .
$$

We have the obvious bound

$$
\begin{aligned}
\frac{1}{n} \sum_{j=1}^{n} B_{2}^{(j)} \leqslant & \frac{1}{v^{2} n^{3}} \sum_{j=1}^{n} \sum_{k \neq l} \mathbf{E}\left|\mathbf{E}\left\{\left(\left(X_{j k}^{(c)}\right)^{2}-\left(\sigma_{j k}^{(c)}\right)^{2}\right)\left(\left(X_{j l}^{(c)}\right)^{2}-\left(\sigma_{j l}^{(c)}\right)^{2}\right) \mid \mathscr{F}^{(j)}\right\}\right| \\
& +\frac{2}{v^{2} n^{3}} \sum_{j=1}^{n} \sum_{k \neq l}\left|\left(\sigma_{j k}^{(c)}\right)^{2}-\sigma^{2}\right| \mathbf{E}\left|\mathbf{E}\left\{\left(\left(X_{j l}^{(c)}\right)^{2}-\left(\sigma_{j l}^{(c)}\right)^{2}\right) \mid \mathscr{F}^{(j)}\right\}\right| \\
& +\frac{1}{n^{3}} \sum_{j=1}^{n} \sum_{k \neq l}\left|\left(\sigma_{j l}^{(c)}\right)^{2}-\sigma^{2}\right|\left|\sigma^{2}-\left(\sigma_{j k}^{(c)}\right)^{2}\right| .
\end{aligned}
$$

In view of the assumptions of Theorem 1.1 we have

$$
\begin{aligned}
\frac{1}{n} \sum_{j=1}^{n} B_{2}^{(j)} & \leqslant C \varepsilon_{n}^{(3)}+C\left(\varepsilon_{n}^{(1)}+\varepsilon_{n}^{(2)}+\mathscr{L}_{n}(\tau)\right) \frac{1}{n} \max _{1 \leqslant j \leqslant n} \sum_{k=1}^{n} \sigma_{j k}^{2} \\
& \leqslant C \varepsilon_{n}^{(3)}+C\left(\varepsilon_{n}^{(1)}+\varepsilon_{n}^{(2)}+\mathscr{L}_{n}(\tau)\right) .
\end{aligned}
$$

Collecting the last inequalities we get

$$
\limsup \frac{1}{n} \sum_{j=1}^{n} \mathbf{E}\left|\eta^{(t, 2)}\right|^{2} \leqslant C \tau^{2}, \quad \lim \sup \frac{1}{n} \sum_{j=1}^{n} \mathbf{E}\left|\eta_{j}^{(t, 4)}\right|^{2} \leqslant C \tau^{2},
$$

where $\eta_{j}^{(t, 4)}=-\sigma^{2} n^{-1}\left(\operatorname{Tr} \mathbf{R}^{(c)}-\mathbf{E} \operatorname{Tr} \mathbf{R}^{(c)}\right)$. The last inequality and inequality (5.2) together imply that $\mathbf{E}\left|n^{-1}(\operatorname{Tr} \mathbf{R}-\mathbf{E} \operatorname{Tr} \mathbf{R})\right|^{2} \rightarrow 0$ uniformly in all compact subset in $\{z=u+i v: v \geqslant 2\}$. As simple implication we obtain that for any $\nu \geqslant 1$ and for $z=u+i v$ with $v>4$

$$
n^{-2} \mathbf{E}\left|\operatorname{Tr} \mathbf{R}^{\nu}-\mathbf{E} \operatorname{Tr} \mathbf{R}^{\nu}\right|^{2} \rightarrow 0 \quad \text { as } n \rightarrow \infty .
$$


To prove this we may use the Cauchy integral formula. We have

$$
\begin{aligned}
\operatorname{Tr} \mathbf{R}^{\nu}-\mathbf{E} \operatorname{Tr} \mathbf{R}^{\nu} & =\frac{1}{(\nu-1) !} \frac{d^{\nu-1}}{d z^{\nu-1}}(\operatorname{Tr} \mathbf{R}-\mathbf{E} \operatorname{Tr} \mathbf{R}) \\
& =\frac{1}{2 i \pi} \oint_{|\zeta-z|=(v-2) / 2} \frac{\operatorname{Tr} \mathbf{R}(\zeta)-\mathbf{E} \operatorname{Tr} \mathbf{R}(\zeta)}{(\zeta-z)^{\nu}} d \zeta .
\end{aligned}
$$

Since $\operatorname{Im}(\zeta-z) \geqslant 2$ we obtain (5.3). We now consider the obvious equality

$$
\frac{1}{x-z}=\frac{1}{x-(z+i V)}+i V \frac{1}{x-(z+i V)} \frac{1}{x-z} \text {. }
$$

This equality yields the following representation:

$$
\begin{aligned}
\operatorname{Tr} \mathbf{R}(z)= & \operatorname{Tr} \mathbf{R}(z+i V)+i V \operatorname{Tr} \mathbf{R}^{2}(z+i V)+\cdots+(i V)^{k} \operatorname{Tr} \mathbf{R}(z+i V) \\
& +(i V)^{k} \operatorname{Tr} \mathbf{R}(z) \mathbf{R}^{k}(z+i V) .
\end{aligned}
$$

Let $v<4$. We take $V=4-v$. Note that

$$
V^{k}\left|\frac{1}{n} \operatorname{Tr} \mathbf{R}(z) \mathbf{R}^{k}(z+i V)\right| \leqslant \frac{(1-v / 4)^{k}}{v} .
$$

We may choose $k$ such that the last term will be less than $\varepsilon$. The variances of the first $k$ terms on the right-hand side of (5.4) tend to zero. This implies that, for any $v>0, n^{-2} \mathbf{E}|\operatorname{Tr} \mathbf{R}(u+i v)-\mathbf{E R}(u+i v)|^{2} \longrightarrow 0$. This completes the proof.

Proposition 5.1. Assume that, for any $v \neq 0$,

$$
\mathscr{R}_{n}(\mathbf{W})(z):=n^{-1} \mathbf{E} \operatorname{Tr}\left(4 \mathbf{I}-\mathbf{W}^{2}\right) \mathbf{R}^{2}(z)+3 n^{-1} \mathbf{E} \operatorname{Tr} \mathbf{W R}(z) \longrightarrow 0
$$

as $n \rightarrow \infty$ uniformly on compact sets in $\mathbf{C} \backslash \mathbf{R}$. Then

$$
\Delta_{n} \rightarrow 0 \quad \text { as } n \rightarrow \infty \text {. }
$$

$\mathrm{P} \mathrm{r}$ o o f. Without loss of generality we consider the case $\operatorname{Im} z>0$ only. In view of relations $(4.1)-(4.5)$ we have

$$
\mathscr{R}_{n}(\mathrm{~W})(z)=\left(z^{2}-4\right) S_{n}^{\prime}(z)-z S_{n}(z)-2,
$$

where $S_{n}(z):=n^{-1} \mathbf{E} \operatorname{Tr}(\mathbf{W}-z \mathbf{I})^{-1}$. Write $\eta_{n}(z):=\mathscr{R}_{n}(\mathbf{W})(z)$. Rewriting the representation (5.5) in integral form we get, for any fixed $z_{0}$ such that $\operatorname{Im} z_{0}>0$,

$$
S_{n}(z)=\frac{\left(z^{2}-4\right)^{1 / 2}}{\left(z_{0}^{2}-4\right)^{1 / 2}}\left(S_{n}\left(z_{0}\right)+\left(z_{0}^{2}-4\right)^{1 / 2} \int_{z_{0}}^{z} \frac{\left(2+\eta_{n}(u)\right) d u}{\left(u^{2}-4\right)^{3 / 2}}\right) .
$$

Let $S(z)$ denote the Stieltjes transform of the semicircle law. According to $(4.5), S(z)$ satisfies the equality

$$
\left(z^{2}-4\right) S^{\prime}(z)-z S(z)-2=0 .
$$

The equations (5.7) and (5.6) together imply that

$S_{n}(z)-S(z)=\frac{\left(z^{2}-4\right)^{1 / 2}}{\left(z_{0}^{2}-4\right)^{1 / 2}}\left(S_{n}\left(z_{0}\right)-S\left(z_{0}\right)\right)+\left(z^{2}-4\right)^{1 / 2} \int_{z_{0}}^{z} \frac{\eta_{n}(\zeta)}{\left(\zeta^{2}-4\right)^{3 / 2}} d \zeta$. 
By definition of the Stieltjes transform, we have, for $z=x+i v$,

$$
\max \left\{\left|S_{n}(z)\right|,|S(z)|\right\} \leqslant v^{-1} \text {. }
$$

Let $\varepsilon>0$. Using (5.9), we choose $z_{0}$ with $\operatorname{Im} z_{0}>2 / \varepsilon$ such that

$$
\left|S_{n}\left(z_{0}\right)-S\left(z_{0}\right)\right| \leqslant \varepsilon \text {. }
$$

Without loss of generality we may assume that $\left|z_{0}^{2}-4\right|>1$.

Let $z$ belong to some compact set $K$ in the upper half-plane. It is easy to check that there exists some constant $C(K)$ depending on this compact set $K$ only such that, for all $n \geqslant 1$, the first summand in (5.8) satisfies the following inequality:

$$
\left|\frac{\left(z^{2}-4\right)^{1 / 2}}{\left(z_{0}^{2}-4\right)^{1 / 2}}\left(S_{n}\left(z_{0}\right)-S\left(z_{0}\right)\right)\right| \leqslant C(K) \varepsilon .
$$

In order to bound the second term on the right-hand side of (5.8) we consider a point $z$ such that $\left|z^{2}-4\right| \geqslant 1$. Let the integral $\int_{z_{0}}^{z}$ denote integration along the any path $\Gamma\left(z_{0}, z\right)$ from $z_{0}$ to $z$ in the upper half-plane. Without loss of generality we assume that, for all $\zeta \in \Gamma\left(z_{0}, z\right)$, the inequality $\left|\zeta^{2}-4\right|>1$ holds. Then there exists a constant $C(K)$ depending on the compact set $K$ only such that

$$
\left|\left(z^{2}-4\right)^{1 / 2} \int_{z_{0}}^{z} \frac{\eta_{n}(\zeta) d \zeta}{\left(\zeta^{2}-4\right)^{3 / 2}}\right| \leqslant C(K) \sup _{\zeta \in \Gamma\left(z_{0}, z\right)}\left|\eta_{n}(\zeta)\right|
$$

If $0<\left|z^{2}-4\right| \leqslant 1$ we have

$$
\left|\int_{z_{0}}^{z} \frac{\eta_{n}(\zeta) d \zeta}{\left(\zeta^{2}-4\right)^{3 / 2}}\right| \leqslant\left|\int_{z_{0}}^{z_{0}^{\prime}} \frac{\eta_{n}(\zeta) d \zeta}{\left(\zeta^{2}-4\right)^{3 / 2}}\right|+\left|\int_{z_{0}^{\prime}}^{z} \frac{\eta_{n}(\zeta) d \zeta}{\left(\zeta^{2}-4\right)^{3 / 2}}\right|
$$

where $z_{0}^{\prime}$ denotes some point in the upper half-plane such that $\left|\left(z_{0}^{\prime}\right)^{2}-4\right|=1$. It is straightforward to check that there exists an absolute constant $C_{1}$ such that, for $\left|z^{2}-4\right| \leqslant 1$,

$$
\left|z^{2}-4\right|^{1 / 2}\left|\int_{z_{0}^{\prime}}^{z}\right| \zeta^{2}-\left.4\right|^{-3 / 2} d \zeta \mid \leqslant C_{1}
$$

The relations (5.7), (5.12), and (5.14) together imply that

$$
\left|z^{2}-4\right|^{1 / 2}\left|\int_{z_{0}}^{z} \frac{\eta_{n}(\zeta) d \zeta}{\left(\zeta^{2}-4\right)^{3 / 2}}\right| \leqslant C(K) \sup _{\zeta \in \Gamma\left(z_{0}, z_{0}^{\prime}\right)}\left|\eta_{n}(\zeta)\right|+C_{1} \sup _{\zeta:\left|z^{2}-4\right| \leqslant 1}\left|\eta_{n}(\zeta)\right|
$$

Furthermore, the relations (5.9)-(5.15) imply that

$$
\limsup _{n \rightarrow \infty}\left|S_{n}(z)-S(z)\right| \leqslant C(K) \varepsilon
$$

uniformly on $z \in K$. That means that $S_{n}(z) \rightarrow S(z)$ as $n \rightarrow \infty$ uniformly on the all compact sets in $\mathbf{C} \backslash \mathbf{R}$. Note that for any distribution function $F(x)$ with Stieltjes transform $T(z)$ we have, for $z=x+i v$,

$$
\operatorname{Im} T(z)=F * F_{v}(x),
$$

where $F_{v}$ denotes Cauchy's distribution function with parameter $v$ and $F_{v}^{\prime}(x)=\pi^{-1} v\left(x^{2}+v^{2}\right)^{-1}$. This concludes the proof. 
6. A truncation of random variables. In this section we prove the following useful statement. Consider some symmetric matrix $\mathbf{D}$ of order $n \times n$. Put

$$
\widetilde{\mathbf{W}}=\mathbf{W}+n^{-1 / 2} \mathbf{D}, \quad \tilde{\mathbf{R}}(z)=(\widetilde{\mathbf{W}}-z \mathbf{I})^{-1} .
$$

Define the Stieltjes transform of the expected spectral distribution function of matrix $\widetilde{\mathbf{W}}$ by

$$
\widetilde{S}_{n}(z)=n^{-1} \mathbf{E} \operatorname{Tr} \widetilde{\mathbf{R}}(z) .
$$

Lemma 6.1. For any $z=u+i v$ with $v>0$ the following inequality holds:

$$
\left|\widetilde{S}_{n}(z)-S_{n}(z)\right| \leqslant n^{-1} v^{-2}\left(\mathbf{E} \operatorname{Tr} \mathbf{D}^{2}\right)^{1 / 2} .
$$

P r o of. Note that

$$
\widetilde{\mathbf{R}}(z)=\mathbf{R}(z)+n^{-1 / 2} \mathbf{R}(z) \mathbf{D} \tilde{\mathbf{R}}(z) .
$$

Denote by $\|\cdot\|_{s}$ the spectral norm of a matrix. For resolvent matrices we have, for $z=u+i v, v>0$,

$$
\max \left\{\|\tilde{\mathbf{R}}(z)\|_{\mathbf{s}},\|\mathbf{R}(z)\|_{\mathbf{s}}\right\} \leqslant v^{-1} .
$$

Inequality (6.2) implies that

$$
n^{-3 / 2}|\operatorname{Tr} \mathbf{R}(z) \mathbf{D} \tilde{\mathbf{R}}(z)| \leqslant n^{-1} v^{-2}\left(\operatorname{Tr} \mathbf{D}^{2}\right)^{1 / 2} .
$$

The relations (6.3) and (6.1) together conclude the proof.

For a random variable $X$, define $X^{(c)}=X \dot{I}_{\{|X| \leqslant c\}}-\mathbf{E} X I_{\{|X| \leqslant c\}}$, which is a truncated and recentred random variable. Introduce the matrix $\mathbf{W}^{(c)}$ with the truncated entries: $W^{(c)}(j, l)=n^{-1 / 2} X_{j l}^{(c)}$. Let $\mathbf{R}^{(c)}(z)=\left(\mathbf{W}^{(c)}-z \mathbf{I}\right)^{-1}$ and $S_{n}^{(c)}(z)=n^{-1} \mathbf{E} \operatorname{Tr} \mathbf{R}^{(c)}(z)$.

Corollary 6.1. For any $z=u+i v$ with $v>0$,

$$
\left|S_{n}^{(c)}(z)-S_{n}(z)\right| \leqslant \frac{1}{v^{2}}\left(\frac{1}{n^{2}} \sum_{j, l=1}^{n} \mathrm{E} X_{j l}^{2} I_{\left\{\left|X_{j l}\right|>c\right\}}\right)^{1 / 2} .
$$

P r o of. The result follows from Lemma 6.1 with $\widetilde{\mathbf{W}}=\mathbf{W}^{(c)}$ and $n^{-1 / 2} \mathbf{D}=\mathbf{W}^{(c)}-\mathbf{W}$.

We define $S_{n}^{(\tau)}(z):=S_{n}^{(\tau \sqrt{n})}(z)$.

Corollary 6.2. For any $z=u+i v$ with $v>0,\left|S_{n}^{(\tau)}(z)-S_{n}(z)\right| \leqslant$ $v^{-2} L_{n}^{1 / 2}(\tau)$.

P r o of. The result follows from Corollary 6.1 with $c=\tau \sqrt{n}$. 
7. The proof of Theorem 1.1. Without loss of generality we may assume that $\sigma^{2}=1$. Recall that, for fixed $\tau>0$,

$$
X_{j l}^{(\tau)}=X_{j l} I_{\left\{\left|X_{j l}\right| \leqslant \tau \sqrt{n}\right\}}-\mathbf{E} X_{j l} I_{\left\{\left|X_{j l}\right| \leqslant \tau \sqrt{n}\right\}} .
$$

Define the symmetric matrix $\mathbf{W}^{(\tau)}$ of order $n \times n$ by $W^{(\tau)}(l, j)=n^{-1 / 2} X_{l j}^{(\tau)}$, for $1 \leqslant l \leqslant j \leqslant n$. Let $\mathbf{R}^{(\tau)}(z)=\left(\mathbf{W}^{(\tau)}-z \mathrm{I}_{n}\right)^{-1}$ and $S_{n}^{(\tau)}(z)=$ $n^{-1} \mathbf{E} \operatorname{Tr} \mathbf{R}^{(\tau)}(z)$. According to Corollary 6.2 we have, for any $z=u+i v$, $v>0$,

$$
\left|S_{n}^{(\tau)}(z)-S_{n}(z)\right| \leqslant v^{-2} L_{n}^{1 / 2}(\tau) .
$$

Note that

$$
S_{n}^{(\tau)^{\prime}}(z)=n^{-1} \mathbf{E} \operatorname{Tr}\left(\mathbf{R}^{(\tau)}\right)^{2} \quad \text { and } \quad S_{n}^{\prime}(z)=n^{-1} \mathbf{E} \operatorname{Tr} \mathbf{R}^{2} .
$$

Using Cauchy's integral formula, we get

$$
\left|S_{n}^{(\tau)^{\prime}}(z)-S_{n}^{\prime}(z)\right| \leqslant 4 v^{-3} L_{n}^{1 / 2}(\tau) .
$$

Introduce the matrices $\mathrm{U}^{(l j)}$, for $1 \leqslant l \leqslant j \leqslant n$, with entries $U^{(l j)}(m, k)=1$ if and only if $\{m, k\}=\{l, j\}$ or $\{m, k\}=\{j, l\}$ and $U^{(l j)}(m, k)=0$ otherwise. Define the matrices $\left(\mathbf{W}^{(\tau)}\right)^{(l j)}=\mathbf{W}^{(\tau)}-n^{-1 / 2} X_{l j} \mathbf{U}^{(l j)}$ and $\left(\mathbf{R}^{(\tau)}\right)^{(l j)}(z)=$ $\left(\left(\mathbf{W}^{(\tau)}\right)^{(l j)}-z \mathbf{I}_{n}\right)^{-1}$. In what follows we shall omit $z$ in the notation of resolvent matrices. The resolvent matrix $R^{(\tau)}$ may be represented as

$$
\begin{aligned}
\mathbf{R}^{(\tau)}= & \left(\mathbf{R}^{(\tau)}\right)^{(l j)}-\frac{1}{\sqrt{n}} X_{l j}^{(\tau)}\left(\mathbf{R}^{(\tau)}\right)^{(l j)} \mathbf{U}^{(l j)}\left(\mathbf{R}^{(\tau)}\right)^{(l j)} \\
& +\frac{1}{n}\left(X_{l j}^{(\tau)}\right)^{2}\left(\mathbf{R}^{(\tau)}\right)^{(l j)} \mathbf{U}^{(l j)} \mathbf{R}^{(\tau)} .
\end{aligned}
$$

For the matrix $\mathbf{R}^{(\tau)}$, notice that

$$
\frac{1}{n} \mathbf{E} \operatorname{Tr} \mathbf{W}^{(\tau)} \mathbf{R}^{(\tau)}=\frac{1}{n \sqrt{n}} \sum_{1 \leqslant j \leqslant l \leqslant n}\left(2-\delta_{j l}\right) \mathbf{E} X_{j l}^{(\tau)} R^{(\tau)}(j, l) .
$$

Using (7.5), we obtain

$$
n^{-1} \mathbf{E} \operatorname{Tr} \mathbf{W}^{(\tau)} \mathbf{R}^{(\tau)}=A_{1}-A_{2}+A_{3},
$$

where

$$
\begin{aligned}
& A_{1}=\frac{1}{n \sqrt{n}} \sum_{1 \leqslant j \leqslant l \leqslant n}\left(2-\delta_{j l}\right) \mathbf{E} X_{j l}^{(\tau)}\left(R^{(\tau)}\right)^{(j l)}(j, l), \\
& A_{2}=\frac{1}{n^{2}} \sum_{1 \leqslant l \leqslant j \leqslant n}\left(2-\delta_{l j}\right) \mathbf{E}\left(X_{l j}^{(\tau)}\right)^{2}\left(R^{(\tau)}\right)^{(l j)} \mathbf{U}^{(l j)}\left(R^{(\tau)}\right)^{(l j)}(l j), \\
& A_{3}=\frac{1}{n^{2} \sqrt{n}} \sum_{1 \leqslant l \leqslant j \leqslant n}\left(2-\delta_{l j}\right) \mathbf{E}\left(X_{l j}^{(\tau)}\right)^{3}\left(R^{(\tau)}\right)^{(l j)} \mathbf{U}^{(l j)} R^{(\tau)}(l, j) .
\end{aligned}
$$

Here and in what follows $\delta_{k j}$ is defined by $\delta_{k j}=1, k=j$, and $\delta_{k j}=0$ otherwise. At first we estimate $A_{3}$. It is easy to see that

$$
\max \left\{\left\|\left(\mathbf{R}^{(\tau)}\right)^{(l j)} \mathbf{U}^{(l j)}\left(\mathbf{R}^{(\tau)}\right)^{(l j)}\right\|_{\mathbf{s}},\left\|\left(\mathbf{R}^{(\tau)}\right)^{(l j)} \mathbf{U}^{(l j)} \mathbf{R}^{(\tau)}\right\|_{\mathbf{8}}\right\} \leqslant v^{-2} .
$$


According to the definition of $X_{l j}^{(\tau)}$ and inequality (7.8), we get

$$
\left|A_{3}\right| \leqslant \frac{2 \tau}{n^{2} v^{2}} \sum_{1 \leqslant l \leqslant j \leqslant n} \mathbf{E}\left(X_{l j}^{(\tau)}\right)^{2} \leqslant \frac{2 \tau}{v^{2}}\left(1+\varepsilon_{n}^{(2)}\right) .
$$

By condition (1.5), $A_{1}=0$. Using relations $(7.8)$ and $\left(\mathbf{R}^{(\tau)}\right)^{(l j)}=\mathbf{R}^{(\tau)}+$ $n^{-1 / 2} X_{j k}^{(\tau)} \mathbf{R}^{(\tau)}{ }^{(l j)} \mathbf{U}^{(l j)} \mathbf{R}^{(\tau)}$, we get

$$
\left|A_{2}^{\prime}-A_{4}\right| \leqslant C\left(1+\varepsilon_{n}^{(2)}\right) v^{-3} \tau,
$$

where

$$
A_{4}=\frac{1}{n^{2}} \sum_{1 \leqslant l \leqslant j \leqslant n}\left(2-\delta_{l j}\right) \mathbf{E}\left(X_{l j}^{(\tau)}\right)^{2} \mathbf{R}^{(\tau)} \mathbf{U}^{(l j)} \mathbf{R}^{(\tau)}(l j) .
$$

A simple calculation shows that

$$
\mathbf{R}^{(\tau)} \mathbf{U}^{(l j)} \mathbf{R}^{(\tau)}(l j)=R^{(\tau)}(j, j) R^{(\tau)}(l, l)+\delta_{l j}\left(R^{(\tau)}\right)^{2}(l, j) .
$$

Substituting (7.12) in (7.11), we get

$$
A_{4}=A_{5}+A_{6}
$$

where

$$
\begin{aligned}
& A_{5}=\frac{1}{n^{2}} \sum_{1 \leqslant l \leqslant j \leqslant n}\left(2-\delta_{l j}\right) \mathbf{E}\left(X_{l j}^{(\tau)}\right)^{2} R^{(\tau)}(j, j) R^{(\tau)}(l, l), \\
& A_{6}=\frac{2}{n^{2}} \sum_{1 \leqslant l<j \leqslant n} \mathbf{E}\left(X_{l j}^{(\tau)}\right)^{2}\left(R^{(\tau)}\right)^{2}(l, j) .
\end{aligned}
$$

Using that $\left|X_{l j}^{(\tau)}\right| \leqslant \tau \sqrt{n}$, we get

$$
\left|A_{6}\right| \leqslant \tau^{2} \frac{1}{n} \sum_{1 \leqslant j \leqslant k \leqslant n}\left(2-\delta_{j k}\right) \mathbf{E}\left|R^{(\tau)}(j, k)\right|^{2} \leqslant 2 \tau^{2} v^{-2} .
$$

For fixed $j=1, \ldots, n$, introduce the symmetric matrix $\mathbf{D}_{j}^{(\tau)}=$ $\left(D_{j}^{(\tau)}(l, k)\right)_{l, k=1}^{n}$, with $D_{j}^{(\tau)}(j, k)=n^{-1 / 2} X_{j k}^{(\tau)}$ if $j \leqslant k \leqslant n, D_{j}^{(\tau)}(k, j)=$ $n^{-1 / 2} X_{k j}^{(\tau)}$ if $1 \leqslant k \leqslant j$, and $D_{j}^{(\tau)}(l, k)=0$, for $1 \leqslant l \leqslant k \leqslant n$, otherwise. Define the matrices $\mathbf{W}^{(\tau, j)}=\mathbf{W}^{(\tau)}-\mathbf{D}_{j}^{(\tau)}$ and $\mathbf{R}^{(\tau, j)}=\left(\mathbf{W}^{(\tau, j)}-z \mathbf{I}_{n}\right)^{-1}$. Using these notation we have, for $j, l=1, \ldots, n$ and $l \neq j$,

$$
R^{(\tau)}(l, l)=R^{(\tau, j)}(l, l)-\mathbf{R}^{(\tau, j)} \mathbf{D}_{j}^{(\tau)} \mathbf{R}^{(\tau)}(l, l)
$$

and

$$
\begin{aligned}
\mathbf{R}^{(\tau, j)} \mathbf{D}_{j}^{(\tau)} \mathbf{R}^{(\tau)}(l, l)= & \frac{1}{\sqrt{n}} \sum_{k=1}^{n} X_{j, k}^{(\tau)} R^{(\tau, j)}(l, j) R^{(\tau)}(k, l) \\
& +\frac{1}{\sqrt{n}} \sum_{k=1}^{n} X_{j, k}^{(\tau)} R^{(\tau, j)}(l, k) R^{(\tau)}(j, l) .
\end{aligned}
$$

Substituting equalities (7.15) and (7.16) in (7.14), we get

$$
A_{5}=A_{7}-A_{8}-A_{9} \text {, }
$$


where

$$
\begin{aligned}
& A_{7}=\frac{1}{n^{2}} \sum_{1 \leqslant l \leqslant j \leqslant n}\left(2-\delta_{l j}\right) \mathbf{E}\left(X_{l j}^{(\tau)}\right)^{2} R^{(\tau)}(j, j) R^{(\tau, j)}(l, l), \\
& A_{8}=\frac{1}{n^{2} \sqrt{n}} \sum_{1 \leqslant l \leqslant j \leqslant n}\left(2-\delta_{l j}\right) \sum_{k=1}^{n} \mathbf{E}\left(X_{l j}^{(\tau)}\right)^{2} X_{j k}^{(\tau)} R^{(\tau, j)}(l, j) R^{(\tau)}(k, l), \\
& A_{9}=\frac{1}{n^{2} \sqrt{n}} \sum_{1 \leqslant l \leqslant j \leqslant n}\left(2-\delta_{l j}\right) \sum_{k=1}^{n} \mathbf{E}\left(X_{l j}^{(\tau)}\right)^{2} X_{j k}^{(\tau)} R^{(\tau, j)}(l, k) R^{(\tau)}(j, l) .
\end{aligned}
$$

Applying (7.5), we obtain

$$
A_{8}=A_{10}-A_{11}-A_{12},
$$

where

$$
\begin{gathered}
A_{10}=\frac{1}{n^{2} \sqrt{n}} \sum_{1 \leqslant l \leqslant j \leqslant n}\left(2-\delta_{l j}\right) \sum_{k=1}^{n} \mathbf{E}\left(X_{l j}^{(\tau)}\right)^{2} X_{j k}^{(\tau)} R^{(\tau, j)}(l, j)\left(R^{(\tau)}\right)^{(j k)}(k, l), \\
A_{11}=\frac{1}{n^{3}} \sum_{1 \leqslant l \leqslant j \leqslant n}\left(2-\delta_{l j}\right) \sum_{k=1}^{n} \mathbf{E}\left(X_{l j}^{(\tau)}\right)^{2}\left(X_{j k}^{(\tau)}\right)^{2} R^{(\tau, j)}(l, k) \\
\quad \times\left(R^{(\tau)}\right)^{(j k)}(j, k) R^{(\tau)}(k, l), \\
A_{12}=\frac{1}{n^{3}} \sum_{1 \leqslant l \leqslant j \leqslant n}\left(2-\delta_{l j}\right) \sum_{k=1}^{n} \mathbf{E}\left(X_{l j}^{(\tau)}\right)^{2}\left(X_{j k}^{(\tau)}\right)^{2} R^{(\tau, j)}(l, k) \\
\times \delta_{j k}\left(R^{(\tau)}\right)^{(j k)}(k, k) R^{(\tau)}(j, l) .
\end{gathered}
$$

It is not difficult to check that

$$
\begin{aligned}
\left|A_{11}\right| & \leqslant \frac{\tau^{2}}{n^{2} v} \sum_{j, k=1}^{n} \mathbf{E}\left(X_{j k}^{(\tau)}\right)^{2} \sum_{l=1}^{n}\left|R^{(\tau)}(k, l) R^{(\tau, j)}(l, k)\right| \\
& \leqslant \frac{\tau^{2}}{n^{2} v} \sum_{j, k=1}^{n} \mathbf{E}\left(X_{j k}^{(\tau)}\right)^{2}\left(\sum_{l=1}^{n}\left|R^{(\tau)}(k, l)\right|^{2}\right)^{1 / 2}\left(\sum_{l=1}^{n}\left|R^{(\tau, j)}(k, l)\right|^{2}\right)^{1 / 2} \\
& \leqslant \frac{\tau^{2}}{n^{2} v^{3}} \sum_{j, k=1}^{n} \mathbf{E}\left(X_{j k}^{(\tau)}\right)^{2} \leqslant \frac{\tau^{2}}{v^{3}}\left(1+\varepsilon_{n}^{(2)}\right)
\end{aligned}
$$

Analogously we obtain the estimate

$$
\left|A_{12}\right| \leqslant \frac{\tau^{2}}{v^{3}}\left(1+\varepsilon_{n}^{(2)}\right) .
$$

By (1.5), we have

$$
A_{10}=\frac{1}{n^{2} \sqrt{n}} \sum_{1 \leqslant l \leqslant j \leqslant n}\left(2-\delta_{l j}\right) \mathbf{E}\left(X_{l j}^{(\tau)}\right)^{3} R^{(\tau, j)}(l, j)\left(R^{(\tau)}\right)^{(j k)}(l, l) .
$$

This equality yields

$$
\left|A_{10}\right| \leqslant \frac{2 \tau}{n^{2} v^{2}} \sum_{1 \leqslant l \leqslant j \leqslant n} \mathbf{E}\left(X_{l j}^{(\tau)}\right)^{2} \leqslant \frac{\tau}{v^{2}}\left(1+\varepsilon_{n}^{(2)}\right) .
$$


Relations (7.18)-(7.22) together imply

$$
\left|A_{8}\right| \leqslant \frac{\tau}{v^{2}}\left(\frac{\tau}{v}+1\right)\left(1+\varepsilon_{n}^{(2)}\right) .
$$

Analogously we get

$$
\left|A_{9}\right| \leqslant \frac{\tau}{v^{2}}\left(\frac{\tau}{v}+1\right)\left(1+\varepsilon_{n}^{(2)}\right) .
$$

Repeating the arguments (7.15)-(7.24) with $R^{(\tau)}(j, j)$ in the definition of $A_{7}$ (see (7.17)), we obtain that

$$
A_{5}=A_{13}+A_{14}
$$

where

$$
A_{13}=\frac{1}{n^{2}} \sum_{1 \leqslant l \leqslant j \leqslant n}\left(2-\delta_{l j}\right) \mathbf{E}\left(X_{l j}^{(\tau)}\right)^{2} R^{(\tau, l)}(j, j) R^{(\tau, j)}(l, l),
$$

and

$$
\left|A_{14}\right| \leqslant \frac{C \tau}{v^{2}}\left(\frac{\tau}{v}+1\right)\left(1+\varepsilon_{n}^{(2)}\right) .
$$

For any $1 \leqslant j<l \leqslant n$, introduce the $\sigma$-algebra $\mathscr{F}\{j\},\{l\}:=\sigma\left\{X_{k m}: 1 \leqslant\right.$ $k \leqslant m \leqslant n$, and $\{k, m\} \cap\{j, l\}=\varnothing\}$. Note that $\mathscr{F}^{\{j\},\{l\}} \subset \mathscr{F}^{(j)}$ and

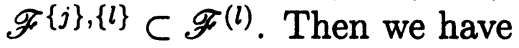

$$
\begin{aligned}
& \mathbf{E}\left(X_{j l}^{(\tau)}\right)^{2} R^{(\tau, l)}(j, j) R^{(\tau, j)}(l, l)=\mathbf{E}\left(\mathbf{E}\left\{\left(X_{j l}^{(\tau)}\right)^{2} R^{(\tau, j)}(l, l) \mid \mathscr{F}^{(l)}\right\} R^{(\tau, l)}(j, j)\right) \\
& =\mathbf{E}\left(\mathbf{E}\left\{\left(X_{j l}^{(\tau)}\right)^{2} R^{(\tau, j)}(l, l) \mid \mathscr{F}^{\{j\},\{l\}}\right\} R^{(\tau, j)}(l, l)\right) \\
& =\mathbf{E}\left(\mathbf{E}\left\{\mathbf{E}\left\{\left(X_{j l}^{(\tau)}\right)^{2} \mid \mathscr{F}^{(j)}\right\} R^{(\tau, j)}(l, l) \mid \mathscr{F}^{\{j\},\{l\}}\right\} R^{(\tau, l)}(j, j)\right) .
\end{aligned}
$$

This equality implies that

$$
\begin{aligned}
& \left|\mathbf{E}\left(X_{j l}^{(\tau)}\right)^{2} R^{(\tau, l)}(j, j) R^{(\tau, j)}(l, l)-\mathbf{E}\left(X_{j l}^{(\tau)}\right)^{2} \mathbf{E} R^{(\tau, l)}(j, j) R^{(\tau, j)}(l, l)\right| \\
& \quad \leqslant \frac{1}{v^{2}} \mathbf{E}\left|\mathbf{E}\left\{\left(X_{j l}^{(\tau)}\right)^{2} \mid \mathscr{F}^{(j)}\right\}-\mathbf{E}\left(X_{j l}^{(\tau)}\right)^{2}\right| .
\end{aligned}
$$

Combining inequalities (7.2), (7.4), (7.17), (7.25), (7.27) and (7.28), we obtain

$$
n^{-1} \mathbf{E} \operatorname{Tr} \mathbf{W}^{(\tau)} \mathbf{R}^{(\tau)}=A_{15}+A_{16},
$$

where

$A_{15}=\frac{1}{n^{2}} \sum_{j, l=1}^{n} \mathrm{E} R^{(\tau, j)}(l, l) R^{(\tau, l)}(j, j), \quad\left|A_{16}\right| \leqslant \frac{1}{n^{2} v^{2}} \sum_{j, l=1}^{n}\left|\sigma_{j l}^{2}-1\right|+\frac{1}{v^{2}} L_{n}(\tau)$.

Consider now $A_{15}$. Applying (7.15) again yields

$$
A_{15}=A_{17}+A_{18}+A_{19}
$$


where

$$
\begin{aligned}
& A_{17}=\frac{1}{n^{2}} \sum_{j, l=1}^{n} \mathbf{E} R^{(\tau, j)}(l, l) R^{(\tau)}(j, j), \\
& A_{18}=\frac{1}{n^{2} \sqrt{n}} \sum_{j, l=1}^{n} \sum_{k=1}^{n} \mathbf{E} X_{l k}^{(\tau)} R^{(\tau, l)}(j, l) R^{(\tau)}(j, k), \\
& A_{19}=\frac{1}{n^{2} \sqrt{n}} \sum_{j, l=1}^{n} \sum_{k=1}^{n} \mathbf{E} X_{l k}^{(\tau)} R^{(\tau, l)}(j, k) R^{(\tau)}(j, l) .
\end{aligned}
$$

For $A_{17}$ we have the following representation:

$$
\begin{aligned}
A_{17} & =\frac{1}{n^{2}} \sum_{j=1}^{n} \mathbf{E}\left[R^{(\tau)}(j, j) \sum_{l=1}^{n} R^{(\tau, j)}(l, l)\right] \\
& =\left(\frac{1}{n} \operatorname{Tr} \mathbf{R}^{(\tau)}\right)^{2}+\frac{1}{n} \sum_{j=1}^{n} \mathbf{E} R^{(\tau)}(j, j)\left(\frac{1}{n} \operatorname{Tr} \mathbf{R}^{(\tau, j)}-\frac{1}{n} \operatorname{Tr} \mathbf{R}^{(\tau)}\right) .
\end{aligned}
$$

Applying Lemma 5.2, we get

$$
A_{17}=\left(\frac{1}{n} \operatorname{Tr} \mathbf{R}^{(\tau)}\right)^{2}+\frac{\theta}{n v}
$$

with some $\theta,|\theta| \leqslant 1$. Furthermore,

$$
\left|A_{18}\right| \leqslant \frac{\tau}{n^{2}} \sum_{k, l=1}^{n} \mathbf{E}\left(\sum_{j=1}^{n}\left|R^{(\tau, l)}(j, l)\right|^{2}\right)^{1 / 2}\left(\sum_{j=1}^{n}\left|R^{(\tau)}(j, k)\right|^{2}\right)^{1 / 2} \leqslant \frac{\tau}{v^{2}}\left(1+\varepsilon_{n}^{(2)}\right) .
$$

Analogously,

$$
\left|A_{19}\right| \leqslant \frac{\tau}{v^{2}}\left(1+\varepsilon_{n}^{(2)}\right) .
$$

Relations (7.28)-(7.34) imply that

$$
\frac{1}{n} \mathbf{E} \operatorname{Tr} \mathbf{W}^{(\tau)} \mathbf{R}^{(\tau)}=-\mathbf{E}\left(\frac{1}{n} \operatorname{Tr} \mathbf{R}^{(\tau)}\right)^{2}+A_{20}
$$

with

$$
\left|A_{20}\right| \leqslant \frac{C \tau}{v^{2}}\left(1+\frac{C \tau}{v}\right)\left(1+\varepsilon_{n}^{(2)}\right) .
$$

Applying Corollary 6.2, we obtain

$$
n^{-1} \mathbf{E} \operatorname{Tr} \mathbf{W}^{(\tau)} \mathbf{R}^{(\tau)}=-\mathbf{E}\left(n^{-1} \operatorname{Tr} \mathbf{R}^{(\tau)}\right)^{2}+A_{21}
$$

with

$$
\left|A_{21}\right| \leqslant \frac{C \tau}{v^{2}}\left(1+\frac{C \tau}{v}\right)\left(1+\varepsilon_{n}^{(2)}\right)+\frac{1}{v^{3}} L_{n}^{1 / 2}(\tau)(1+|z|) .
$$

Here we have used the equality

$$
\mathbf{W R}=z \mathbf{R}+\mathbf{I} .
$$


Finally, by Lemma 5.1, we obtain

$$
n^{-1} \mathbf{E} \operatorname{Tr} \mathbf{W R}=-\left(\mathbf{E} n^{-1} \operatorname{Tr} \mathbf{R}\right)^{2}+A_{22},
$$

where $\left|A_{22}\right| \leqslant\left|A_{21}\right|+\delta_{n}$ and $\delta_{n} \rightarrow 0$ as $n \rightarrow \infty$.

Note that

$$
\frac{1}{n} \mathbf{E} \operatorname{Tr} \mathbf{W R}^{2}(z)=-\frac{d}{d z} \mathbf{E} \operatorname{Tr} \mathbf{W R}(z) .
$$

Applying Cauchy's integral formula, we obtain

$$
n^{-1} \mathbf{E} \operatorname{Tr} \mathbf{W R}^{2}(z)=-2\left(\mathbf{E} n^{-1} \operatorname{Tr} \mathbf{R}^{2}\right)\left(\mathbf{E} n^{-1} \operatorname{Tr} \mathbf{R}\right)+A_{23},
$$

where

$$
\left|A_{23}\right| \leqslant \frac{C \tau}{v^{3}}\left(1+\frac{C \tau}{v}\right)\left(1+\varepsilon_{n}^{(2)}\right)+\frac{C}{v^{4}} L_{n}^{1 / 2}(\tau)(1+|z|) .
$$

Furthermore,

$$
\begin{aligned}
& n^{-1} \mathbf{E} \operatorname{Tr}\left(4 \mathbf{I}_{n}-\mathbf{W}^{2}\right) \mathbf{R}^{2}(z)+3 n^{-1} \mathbf{E} \operatorname{Tr} \mathbf{W R}(z) \\
& \quad=4 n^{-1} \mathbf{E} \operatorname{Tr} \mathbf{R}^{2}(z)-z n^{-1} \mathbf{E} \operatorname{Tr} \mathbf{W} \mathbf{R}^{2}(z)+2 n^{-1} \mathbf{E} \operatorname{Tr} \mathbf{W R}(z) .
\end{aligned}
$$

Substituting (7.40) and (7.41) in (7.43), we get

$$
\begin{gathered}
n^{-1} \mathbf{E} \operatorname{Tr}\left(4 \mathbf{I}-\mathbf{W}^{2}\right) \mathbf{R}^{2}(z)+3 n^{-1} \mathbf{E} \operatorname{Tr} \mathbf{W R}(z) \\
=4 S_{n}^{\prime}(z)+2 z S_{n}^{\prime}(z) S_{n}(z)-2 S_{n}^{2}(z)+A_{24},
\end{gathered}
$$

where

$$
\left|A_{24}\right| \leqslant \frac{C \tau}{v^{3}}\left(1+\frac{C \tau}{v}\right)\left(1+\varepsilon_{n}^{(2)}\right)+\frac{C}{v^{4}} L_{n}^{1 / 2}(\tau)\left(1+|z|^{2}\right) .
$$

Relation (7.38) and (7.39) together imply that $S_{n}^{2}(z)+z S_{n}(z)+1=A_{25}$, with

$$
\left|A_{25}\right| \leqslant \frac{C \tau}{v^{3}}\left(1+\frac{C \tau}{v}\right)\left(1+\varepsilon_{n}^{(2)}\right)+\frac{C}{v^{4}} L_{n}^{1 / 2}(\tau)\left(1+|z|^{2}\right) .
$$

The last equality may be rewritten as

$$
2+z S_{n}(z)=-S_{n}(z)\left(z+2 S_{n}(z)\right)+2 A_{25} .
$$

Relation (7.44) and (7.46) together imply

$$
\begin{aligned}
& n^{-1} \mathbf{E} \operatorname{Tr}\left(4 \mathrm{I}-\mathbf{W}^{2}\right) \mathbf{R}^{2}(z)+3 n^{-1} \mathbf{E} \operatorname{Tr} \mathbf{W R}(z) \\
& \quad=-2 S_{n}^{\prime}(z) S_{n}(z)\left(z+2 S_{n}(z)\right)-2 S_{n}^{2}(z)+A_{26},
\end{aligned}
$$

where

$$
\left|A_{26}\right| \leqslant \frac{C \tau}{v^{3}}\left(1+\frac{C \tau}{v}\right)\left(1+\varepsilon_{n}^{(2)}\right)+\frac{C}{v^{4}} L_{n}^{1 / 2}(\tau)\left(1+|z|^{2}\right) .
$$

Note that

$$
S_{n}^{\prime}(z)\left(z+2 S_{n}(z)\right)+S_{n}(z)=\frac{d}{d z}\left\{S_{n}^{2}+z S_{n}(z)+1\right\} .
$$


Applying Cauchy's integral formula, we get

$\left|S_{n}^{\prime}(z)\left(z+2 S_{n}(z)\right)+S_{n}(z)\right| \leqslant \frac{C \tau}{v^{3}}\left(1+\frac{C \tau}{v}\right)\left(1+\varepsilon_{n}^{(2)}\right)+\frac{C}{v^{4}} L_{n}^{1 / 2}(\tau)\left(1+|z|^{2}\right)$.

Finally, using that $\left|S_{n}(z)\right| \leqslant v^{-1}$, we obtain

$$
\begin{aligned}
& \left|n^{-1} \mathbf{E} \operatorname{Tr}\left(4 \mathbf{I}-\mathbf{W}^{2}\right) \mathbf{R}^{2}(z)+3 n^{-1} \mathbf{E} \operatorname{Tr} \mathbf{W R}(z)\right| \\
& \quad \leqslant \frac{C \tau}{v^{4}}\left(1+\frac{C \tau}{v}\right)\left(1+\varepsilon_{n}^{(2)}\right)+\frac{C}{v^{4}} L_{n}^{1 / 2}(\tau)\left(1+|z|^{2}\right) .
\end{aligned}
$$

From relation (7.48) it follows that

$$
\lim _{n \rightarrow \infty}\left|n^{-1} \mathbf{E} \operatorname{Tr}\left(4 \mathbf{I}-\mathbf{W}^{2}\right) \mathbf{R}^{2}(z)+3 n^{-1} \mathbf{E} \operatorname{Tr} \mathbf{W R}(z)\right|=0,
$$

uniformly on all compacts in $\mathbf{C} \backslash \mathbf{R}$. Applying Proposition 5.1 concludes the proof.

\section{REFERENCES}

1. Bai $Z$. D. Methodologies in spectral analysis of large-dimensional random matrices: a review. - Statist. Sinica, 1999, v. 9, № 3, p. 611-661.

2. Bronk $B . V$. Topics in the theory of random matrices. Thesis. Princeton: Princeton Univ., 1964.

3. Гирко В.Л. Асимптотика распределения спектра случайных матриц. - Успехи матем. наук, 1989 , т. 44, № 4, с. 7-34.

4. Götze F., Tikhomirov A.N. Rate of convergence to the semicircle law. - Probab. Theory Related Fields, 2003, v. 127, № 2, p. 228-276.

5. Градитейн И. С., Рыжик И. М. Таблицы интегралов, сумм, рядов и произведений. М.: Наука, 1971, 1108 c.

6. Хорн Р., Джсосон Ч. Матричный анализ. М.: Мир, 1989, 655 с.

7. Khorunzhy A.M., Khoruzhenko B. A., Pastur L. A. Asymptotic properties of large random matrices with independent entries. - J. Math. Phys., 1996, v. 37, № 10, p. 5033-5060.

8. Марченко В. А., Пастур Л. А. Распределение собственных значений в некоторых ансамблях случайных матриц. - Матем. сб., 1967, т. 72, № 4, с. 507-536.

9. Mehta M. L. Random Matrices. Boston: Academic Press, 1991, 562 p.

10. Pastur L. A., Shcherbina $M$. Universality of the local eigenvalue statistics for a class of unitary invariant random matrix ensembles. - J. Statist. Phys., 1997, v. 86, № 1-2, p. 109-147.

11. Boutet de Monvel A., Pastur L., Shcherbina M. On the statistical mechanics approach in the random matrix theory: the integrated density of states. - J. Statist. Phys., 1995 , v. 79 , № 3-4, p. 585-611.

12. Pastur $L$. Eigenvalue distribution of random matrices: some recent results. - Ann. Inst. H. Poincaré Phys. Théor., 1996, v. 64, p. 325-337.

13. Пастур Л. А. Спектры случайных самосопряженных операторов. - Успехи матем. наук, 1973 , т. 28 , № 1, с. 3-64.

14. Rosenzweig N. Statistical mechanics of equally likely quantum systems. - Statistical Physics (Brandeis Summer Institute, 1962, v. 3). New York: W. A. Benjamin, 1963, p. 91-158.

15. Wigner E. P. Characteristic vectors of bordered matrices with infinite dimensions. Ann. Math., 1955 , v. 62 , p. $548-564$.

16. Wigner E. P. On the distribution of the roots of certain symmetric matrices. - Ann. Math., 1958 , v. 67 , p. $325-327$. 